\title{
A Multimodal Serious-Game to Teach Fractions in Primary School
}

\author{
Simone Ghisio \\ Universitá di Genova, Casa Paganini, \\ DIBRIS \\ Genova, Italy, Italy \\ simoneghisio@gmail.com
}

\author{
Paolo Alborno \\ Universitá di Genova, Casa Paganini, \\ DIBRIS \\ Genova, Italy, Italy \\ paoloalborno@gmail.com
}

\author{
Erica Volta \\ Universitá di Genova, Casa Paganini, \\ DIBRIS \\ Genova, Italy, Italy \\ erica.volta@edu.unige.it
}

\author{
Monica Gori \\ Fondazione Istituto Italiano di \\ Tecnologia \\ Genova, Italy, Italy \\ monica.gori@iit.it
}

\author{
Gualtiero Volpe \\ Universitá di Genova, Casa Paganini, \\ DIBRIS \\ Genova, Italy, Italy \\ gualtiero.volpe@unige.it
}

\begin{abstract}
Multisensory learning is considered a relevant pedagogical framework for education since a very long time and several authors support the use of a multisensory and kinesthetic approach in children learning. Moreover, results from psychophysics and developmental psychology show that children have a preferential sensory channel to learn specific concepts (spatial and/or temporal), hence a further evidence for the need of a multisensory approach. In this work, we present an example of serious game for learning a particularly complicated mathematical concept: fractions. The main novelty of our proposal comes from the role covered by the communication between sensory modalities in particular, movement, vision, and sound. The game has been developed in the context of the EU-ICTH2020 weDRAW Project aiming at developing new multimodal technologies for multisensory serious-games on mathematical concepts for primary school children.
\end{abstract}

\section{CCS CONCEPTS}

- Human-centered computing $\rightarrow$ Empirical studies in HCI; Laboratory experiments;

\section{KEYWORDS}

Multisensory learning, multimodal technologies, serious games

\section{ACM Reference Format:}

Simone Ghisio, Paolo Alborno, Erica Volta, Monica Gori, and Gualtiero Volpe. 2017. A Multimodal Serious-Game to Teach Fractions in Primary School. In Proceedings of 1st ACM SIGCHI International Workshop on Multimodal Interaction for Education (MIE'17). ACM, New York, NY, USA, 4 pages. https: //doi.org/10.1145/3139513.3139524

Permission to make digital or hard copies of part or all of this work for personal or classroom use is granted without fee provided that copies are not made or distributed for profit or commercial advantage and that copies bear this notice and the full citation on the first page. Copyrights for third-party components of this work must be honored For all other uses, contact the owner/author(s).

MIE'17, November 13, 2017, Glasgow, UK

(c) 2017 Copyright held by the owner/author(s)

ACM ISBN 978-1-4503-5557-5/17/11.

https://doi.org/10.1145/3139513.3139524

\section{INTRODUCTION}

\subsection{Background}

Literature shows a vast agreement about multisensory learning and its benefit in education contexts [7], [18], [17], but, despite this, current technologies do not still sufficiently include and take into account this evidence and the same can be argued on the game design for education. Furthermore, at school quite all the teaching is conducted using almost exclusively the visual channel, leaving quite no space for the other sensory modalities. However, recent scientific research states that the visual channel is not always the more powerful and effective perceptive channel for learning, as we can read in [13] and [11] showing that children have a preferential sensory channel to learn specific concepts (spatial and/or temporal). Technology has been widely developed to include sensors and mobile devices for embodied interaction in learning, however none of them sufficiently exploited multisensory integration for teaching, grounding on these psychophysics evidences. In this work, we present technological solutions for enabling an innovative methodology for education, involving multisensory and adaptive learning supported by a game integration. The idea is to find, through psychological experiments, the sensory modality that suits best according to the child's needs and the concepts to be taught, and to use this modalities in a game environment to train her. In other words, our approach exploits multisensory feedback starting from a full-body movement interaction. Furthermore, we base our approach on music, as an effective medium to understand algebraic concepts. The idea of mediating learning through performing arts is not completely new and there is some evidence from neuroscience and cognitive science that stress the positive role of music in the educational context. Besides, there is a quite immediate association between music and arithmetic, based on the suggestion that temporal sequences are at the base of rhythm and that there is an immediate relation between music elements and numerosity. One of the major contribution of games and applications that exploit multisensory feedback is to provide the same opportunities to both typical and impaired children (e.g., sensory modalities which are impaired can be replaced by other modalities), helping teachers in their educational work and in creating easily a positive inclusion experience in the class. 


\section{UNDERSTANDING FRACTIONS THROUGH THE BODY}

Besides the traditional school teaching where learning is primarily based on the visual channel, in our prototype arithmetic is naturally linked to music expression; there are, in fact, numerous elements, i.e., pitch, melody, rhythm, tempo and more on, that are in relation with numbers and fractions and this link is recognized since ancient times. Arithmetic (as geometry) is also largely based on symbolic representation. However, if we change the learning perceptive channel, or we associate more channels together, we do not change necessarily the way of learning. For this reason, in this prototype we use not only a multisensory framework, but we associate a multimodal experience with a full-body interaction in an active learning approach to create knowledge acquired by doing and stored as a motor response. In this paper, we focus on a particular teaching element: fractions. Fractions are a struggling concept for children to be learnt. Particularly, the problems to face are:

- Understanding the relation between the 'whole'and the 'unit' in fractions, both for typical and visually impaired children.

- Equivalences between fractions (e.g., $\frac{2}{4}$ and $\frac{1}{2}$ ).

- Understanding the relation between the denominator of the fraction and the unit, so that having a larger number as a denominator does not mean to have a bigger fraction but a smaller one.

- Fractions that exceed the unit.

- The relation between fractions and percentage, particularly for visually impaired children, that do not have the practical experience of what percentages are and where and how they are used.

In literature, we can find a quite general consensus on using positively music to teach mathematical concepts and enhance spatialtemporal reasoning or reading abilities, e.g., see [9], [12], [16], [15]. Music training, in fact, can improve phonological deficits that can cause dyslexia in children [14] and it has been discovered that six months of music training can positively affect spatial-temporal reasoning [5]. Moreover, students that have private music lessons perform significantly better on mathematics than students who did not have music lessons, with a positive relevance for students who play keyboards [10].

Another interesting suggestion we received from previous research, is the special relation between music and mathematics related to rhythmic pattern. Moreover, it can be proved that by working on music rhythmic elements, a quite natural learning is obtained about arithmetic elements such as the fractions related to those rhythmic elements [1]. Identifying and using a variety of rhythmic patterns, in fact, helps children to develop the ability to better organize their information and relating these patterns to numbers and measurements helps them to understand the connection to mathematical topics.

In the game prototype we present, we take into account all the interdisciplinary suggestions we mentioned here. We design, then, the Fraction Body game, based on the insight that we can see our body as the body of a fraction, where the upper limbs represent the numerator and the lower limbs the denominator. The child can explore different kind of fractions, receiving an audio-visual feedback, and interacting through the body with the system.

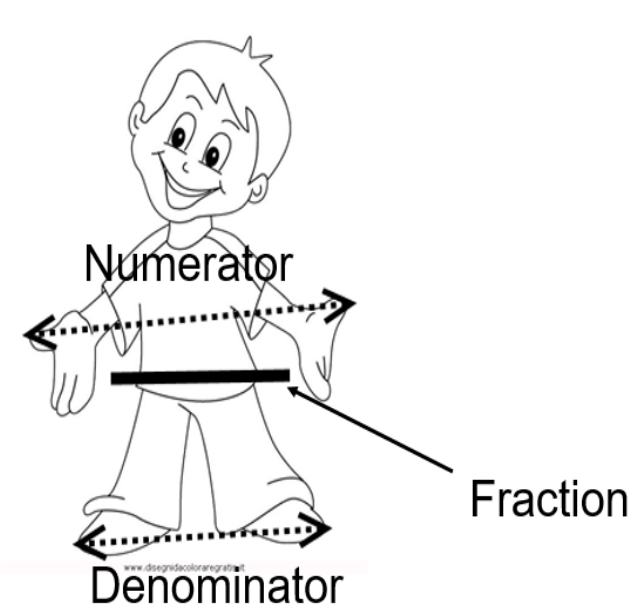

Figure 1: Sketch of the prototyped full-body interaction.

\subsection{Design and Architecture}

The game has been developed in the context of the weDRAW project. Its implementation is based on the EyesWeb XMI software platform [4], [3], [2]. EyesWeb is a software platform used in several applications for real-time dance, music, and rehabilitation and multimedia apps.

In this particular implementation, the developed application maps body movements onto video and audio, to let the children experience multisensory learning, understanding the connection between proprioceptive feedback and feedback from the other modalities, creating and understanding the mentioned association between arithmetic and music. Furthermore, it has also been demonstrated that interactive sonification and multimodal experience can effectively improve cognitive abilities and learning processes [6], [8]. The game we propose is used in the classroom and its set-up consists of a Kinect V2, connected to a PC, using the EyesWeb XMI platform [4] to manage the development, execution and evaluation of the serious game.

One of the major difficulties, as we mentioned above, is to design a clear relation between the 'whole'and the 'unit'used as reference, in all sensory modalities, to make the same concept of fraction understandable for everyone, despite cognitive, learning or sensory impairment.

The serious-game we present is designed as follows:

- The interaction is based on child's limbs movement tracked with Kinect V2;

- The game is designed to be played by one child at time and the feedback provided is an audio/video feedback;

- The game UI is designed using Unity and connected to EyesWeb XMI.

The game has the following features:

- It has a multilevel structure to be suitable for each child, according to learning target and individual needs; 

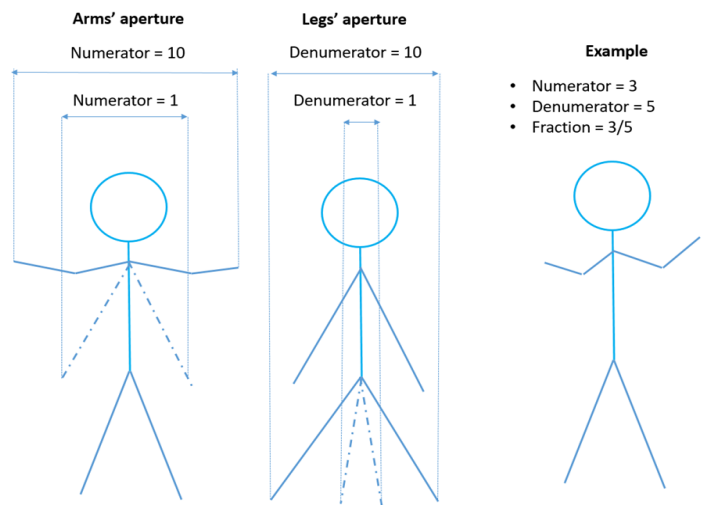

Figure 2: A game session example

- During a gaming session, movement data are stored in order to perform analysis and compute evaluation on child's performance and progression.

\subsection{Game Session}

Our pedagogical target is to teach children the concept of fractions in a general way i.e., the relation between the fraction and the unit and between different types of fractions. We design and propose a game based on the metaphor of the body as a fraction representation. When the game starts, the child can explore different types of fraction moving her/his limbs to create the numerator (upper limbs) and denominator (lower ones) of the fraction and listening to the different rhythmic pattern produced (see Figure 1). Child's arms and legs aperture is measured and mapped to sound. Aperture is detected by the system and internally quantized in the following interval: $[1,10]$. The game interface will ask the player to reproduce a randomly generated fraction, displaying a numerator and a denominator, e.g., $\frac{3}{10}$. The child try to reproduce by moving her/his arms and legs (widening and resting) guided by the auditory feedback, until the desired required fraction is reached. The auditory feedback we propose is very simple: a "beep" with a default pitch and frequency.

The frequency is measured in bpm (beat per minute). When the arms and leg aperture are equivalent, the system recognize a unit (e.g., $\frac{3}{3}$ ) then the typical heart beat rate is mapped into the sound model, i.e., $60 \mathrm{bpm}$. If the numerator is larger than the denominator, two changes are applied to the auditory feedback:

- bpm is increased;

- pitch is increased;

The maximum bpm and the highest pitch are reached if the player maintains a very small amplitude in the arms equivalent to number 1, and very large amplitude on legs, equivalent to 10.

In addition to the rhythmic feedback, the system presents a visual representation of the fraction and an audio/visual comment helpful to link the fraction the child made with the body and experienced as an audiovisual stimulation, to its specific symbolic arithmetic representation (e.g., $\frac{1}{2}, \frac{1}{4}$ and so on). This last point is particularly relevant from an educational point of view, since as teachers and educators explained us, one of the difficulties in understanding fractions for children is to understand the relation between the quantities itself and how that quantity is symbolically represented in arithmetic.

\section{CONCLUSION AND FUTURE WORK}

The game we propose is a first prototype of a multilayer serious game to teach arithmetic concepts. The pre- and post-test to study the efficacy of this methodology and design will be conducted starting from September in primary schools both in Italy and in the UK. Based on the results we will collect, we will extend the game grounding on the evidence we will have from psychophysics and pedagogical and technical areas, to better design the multimodal and multisensory full-body interaction and game experience.

\section{ACKNOWLEDGMENTS}

We thank all the primary schools in Genova (Italy) and in London (UK) that have participated in this first phase of the project, specifically in the sessions with the pedagogues to underlie the key concepts we have focused on, and as in the design session with us to sketch an interaction that can concretely help children to better learn arithmetic. We also thank Istituto Chiossone for their help in understanding the needs of visually impaired children. weDRAW has received funding from the European Union's Horizon 2020 Research and Innovation Program under Grant Agreement No.732391.

\section{REFERENCES}

[1] 1998. The Rhythm of Mathematics. Classroom Compass - Eisenhowever Southwest Consortium for the Improvement of Mathematics and Science Teaching 4, 2 (1998).

[2] Camurri A., Mazzarino B., and Volpe G. 2004. Expressive Interfaces. Cognition Technology and Work 6, 1 (2004), 15-22.

[3] Camurri A., Mazzarino B., Ricchetti M., Timmers R., and Volpe G. 2004. Multimodal analysis of expressive gesture in music and dance performance. In GestureBased Communication in Human-Computer Interaction, Springer Berlin Heidelberg (Ed.). 20-39.

[4] Camurri A., Hashimoto S., Richetti M., Ricci A., Suzuki K., Trocca R., and Volpe G. 2000. EyesWeb: Toward Gesture and Affect Recognition in Interactive Dance and Music Systems. Computer Music fournal (2000), 57-69.

[5] Graziano A. B., Peterson M., and Shaw G. L. 1999. Enhanced learning of proportional math through music training and spatial-temporal training. Neurological research 21, 2 (1999), 139-152.

[6] Dubus G. and Bresin R. 2013. A Systematic Review of Mapping Strategies for the Sonification of Physical Quantities. PLoS ONE 8, 12 (2013).

[7] Fernald G. 1921. The effect of kinesthetic factors in development of word recognition in the case of non-readers. Fournal of Educational Research 4 (1921).

[8] Varni G. and Dubus G. et al. 2012. Interactive sonification of synchronisation of motor behaviour in social active listening to music with mobile devices. Fournal on Multimodal User Interfaces 5 (2012), 157-173.

[9] Rauscher F. H., Shaw G. L., Levine L. J., Wright E. L., Dennis W. R., and Newcomb R. L. 1997. Music training causes long-term enhancement of preschool children's spatial-temporal reasoning. Neurological Research 19 (1997), 2-8.

[10] Cheek J.M. and L.R. Smith. 1999. Music training and Mathematics achievement. Adolescence 34, 136 (1999), 759-761.

[11] Barrett M. and Newell F. 2015. Task- Specific, Age Related Effects in the CrossModal Identification and Localisation of Objects. Multisensory research 28, 1-2 (2015), 111-151.

[12] Forgeard M., Gottfried S., Andrea N., Camilla R., Udita I., and Ellen W. 2008. The relation between music and phonological processing in normal-reading children and children with dyslexia. Music Perception: An Interdisciplinary fournal 25, 4 (2008), 383-390.

[13] Gori M., Del Viva M., Sandini G., and Burr D. C. 2008. Young children do not integrate visual and haptic form information. Current Biology 18, 9 (2008), 694-8.

[14] Habib M., Lardy C., Desiles T., Commeiras C., Chobert J., and Besson Mireille. 2016. Music and Dyslexia: A New Musical Training Method to Improve Reading and Related Disorders. Frontiers in Psychology 7, 26 (2016). 
[15] Piro J. M. and Ortiz C. 2009. The effect of piano lessons on the vocabulary and verbal sequencing skills of primary grade students. Psychology of Music 37, 3 (2009), 325-347.

[16] Claudia S. Salcedo. 2010. The Effects Of Songs In The Foreign Language Classroom On Text Recall, Delayed Text Recall And Involuntary Mental Rehearsal. Journal of College Teaching and Learning 7, 6 (2010), 19-30.

[17] A. Strauss and L. E. Lehtinen. 1947. Psychopathology and Education of the BrainInjured Child. Grune and Stratton.

[18] Orton S. T. 1928. Specific reading disability - strephosymbolia. Journal of American Medical Association 90 (1928), 1095-1099. 